\title{
LM-01K038
}

May 8, 2001

\section{Primary Water SCC Understanding and Characterization Through Fundamental Testing in the Vicinity of the Nickel/Nickel Oxide Phase Transition}

D.S. Morton, S.A. Attanasio, G.A. Young

\section{NOTICE}

This report was prepared as an account of work sponsored by the United States Government. Neither the United States, nor the United States Department of Energy, nor any of their employees, nor any of their contractors, subcontractors, or their employees, makes any warranty, express or implied, or assumes any legal liability or responsibility for the accuracy, completeness or usefulness of any information, apparatus, product or process disclosed, or represents that its use would not infringe privately owned rights. 


\title{
Primary Water SCC Understanding and Characterization Through Fundamental Testing in the Vicinity of the Nickel/Nickel Oxide Phase Transition
}

\author{
D.S. Morton, S.A. Attanasio and G.A. Young \\ Lockheed Martin \\ P.O. Box 1072 \\ Schenectady, NY 12301
}

\begin{abstract}
This paper quantifies the nickel alloy stress corrosion crack growth rate (SCCGR) dissolved hydrogen level functionality. SCCGR has been observed to exhibit a maximum in proximity to the nickel/nickel oxide phase transition. The dissolved hydrogen level SCCGR dependency has been quantified in a phenomenological model in terms of the stability of nickel oxide not the dissolved hydrogen level. The observed SCCGR dependency has been extended to lower temperatures through the developed model and Contact Electrical Resistance (CER) measurements of the nickel/nickel oxide phase transition. Understanding obtained from this hydrogen level SCC functionality and complementary SCC subprocesses test results is discussed. Specifically, the possible SCC fundamental subprocesses of corrosion kinetics, hydrogen permeation and pickup have also been measured for nickel alloys. Secondary Ion Mass Spectroscopy (SIMS) analysis has been performed on SCCGR specimens tested in heavy water $\left(\mathrm{D}_{2} \mathrm{O}\right)$.
\end{abstract}

\section{INTRODUCTION}

Hydrogen is often added to high temperature water to maintain low levels of dissolved oxygen and thereby minimize corrosion of structural metals. Numerous studies ${ }^{1-7}$ have shown that hydrogen dissolved in high temperature water $\left(288\right.$ to $\left.360^{\circ} \mathrm{C}\right)$ or hydrogen gas in steam $\left(400^{\circ} \mathrm{C}\right)$ affects the stress corrosion cracking (SCC) performance of nickel alloys (e.g., alloys 600 and $\mathrm{X}-750$ ). Test results have demonstrated that a crack growth rate maximum, with respect to coolant hydrogen variation, is observed in proximity to a key phase transition, the nickel $(\mathrm{Ni})$ to nickel oxide (NiO) phase transition, see Figure 1.

Reference (1) identified that the SCC hydrogen dependency is fundamentally described by the extent that the alloy's corrosion potential deviates from the potential of the $\mathrm{Ni} / \mathrm{NiO}$ phase transition. This potential difference represents the relative stability of the SCC controlling oxide films (e.g., crack tip oxides are often of a $\mathrm{NiO}$ structure ${ }^{8}$ ).

This paper reports results from numerous tightly controlled SCC crack growth rate (SCCGR) tests as a function of dissolved hydrogen level for three nickel alloys (X-750 AH, X-750 HTH and alloy 600). Except for new tests of alloy 600 at a stress intensity factor of $65.9 \mathrm{MPa} / \mathrm{m}$, these tests are a direct continuation of the Reference (1) effort. A phenomenological SCCGR correlation has been developed to describe the observed nickel alloy SCC dependency on dissolved hydrogen. This hydrogen SCC model, in association with Contact Electrical Resistance (CER) measurements ${ }^{9}$ of the $\mathrm{Ni} / \mathrm{NiO}$ phase transition from 288 to $360^{\circ} \mathrm{C}$ permits the ability to extrapolate the SCCGR hydrogen functionality to lower temperatures.

\section{EXPERIMENTAL PROCEDURE}

The nickel alloys investigated in this study were mill-annealed alloy 600 , alloy X-750 condition HTH, and alloy X750 condition AH. The compositions of these alloys have been provided in Reference (1). Alloy 600 was fabricated into standard $25.4 \mathrm{~mm}$ compact tension (CT) specimens with $10 \%$ side grooves and alloy X-750 was fabricated into standard $10.2 \mathrm{~mm}$ CT specimens. All specimens were fabricated in a longitudinal-transverse (LT) orientation. Test specimens were air fatigue precracked, to a nominal a/W of 0.5 for alloys 600 and $\mathrm{X}-750 \mathrm{AH}$ and 0.45 for alloy X$750 \mathrm{HTH}$, using the fatigue precracking procedure from ASTM E399 Annex 2. The alloy X-750 tests were conducted under pure constant load test conditions whereas a $10 \%$ daily unload was performed in the alloy 600 tests. 
The initial nominal stress intensity factor $\left(\mathrm{K}_{\mathrm{I}}\right)$ in the alloy X-750 AH tests was $27.5 \mathrm{MPa} \sqrt{\mathrm{m}}$ and $49.4 \mathrm{MPa} / \mathrm{m}$ for the alloy X-750 HTH tests. Alloy 600 tests were conducted at $K_{I}$ levels of 27.5 and $65.9 \mathrm{MPa} V_{\mathrm{m}}{ }^{(1)}$.

Constant load stress corrosion crack growth rate (SCCGR) tests were conducted in active load servoelectric machines with digital control electronics monitored through a computerized data acquisition system. A description of the recirculating high flow rate autoclave system has previously been described. ${ }^{10}$ Crack lengths were monitored in-situ using electrical potential drop (EPD) according to ASTM E647, Annex 3, and were verified by post-test destructive examinations (DE). Alloy 600 EPD measured active voltages (proportional to crack extension) were normalized for alloy 600 resistivity changes measured in an unloaded non-precracked $10.2 \mathrm{~mm}$ CT specimen of the same heat. Since alloy X-750 resistivity does not change appreciably with time ${ }^{11}, \mathrm{X}-750$ EPD resistivity normalization was not performed in tests with these materials.

\section{Test Environment}

The SCCGR test chemistries were deaerated high purity water with various levels of dissolved hydrogen. The feed tank solution was buffered to a room temperature $\mathrm{pH}$ of 10.1 to maintain a near neutral autoclave $\mathrm{pH}$ at the test temperature. Separate tests were performed at multiple hydrogen concentrations for each alloy (between 1 and 149 $\mathrm{cc} / \mathrm{kg}$ ). The desired hydrogen concentrations were obtained by varying the feed tank hydrogen overpressure according to Henry's law. A room temperature Henry's law coefficient of $5.9 \mathrm{kPa} /(\mathrm{cc} / \mathrm{kg})$ was employed. Cylinders of mixed gas containing $4 \%$ or $14.7 \%$ hydrogen, with the balance argon, were used to obtain hydrogen levels less than $20 \mathrm{cc} / \mathrm{kg}$. Pure nitrogen gas was employed to obtain test conditions with very low hydrogen levels ${ }^{(2)}$. Since the autoclave turnover rate is relatively rapid (once every hour), autoclave effluent dissolved gas levels are analogous to feed tank levels, and the oxygen levels for all tests were $<10 \mathrm{ppb}$.

Alloys 600 and X-750 AH tests were conducted at $338^{\circ} \mathrm{C}$ and alloy X-750 HTH tests were conducted at $360^{\circ} \mathrm{C}$. Prior to the start of the alloys 600 and X-750 AH SCC tests, test specimens were prefilmed in the load train for 10 and 14 days, respectively, at $\mathrm{K}_{\mathrm{I}} \cong 4 \mathrm{MPa} \sqrt{\mathrm{m}}$ and $260^{\circ} \mathrm{C}$ at the hydrogen level of the SCC test. Alloy X-750 $\mathrm{HTH}$ specimens were not prefilmed. The differences in material prefilming procedures were a consequence of historical testing practices.

Trends in $\mathrm{pH}$ and contaminant levels were monitored through system in-line conductivity probes. Anion contaminant levels were verified as less than detectable $(\sim 25 \mathrm{ppb})$ by ion chromatography (IC) analysis of autoclave effluent samples. Autoclave cation contaminants were monitored through analysis of autoclave effluent samples by inductively coupled plasma (ICP) spectroscopy. Cation contaminant levels typically coincided with cation solubility limits (e.g., $\sim 2 \mathrm{ppb} \mathrm{Fe}$ ) at temperature.

Upon completion of SCC testing, alloy 600 and X-750 AH test specimens were heat-tinted at $454^{\circ} \mathrm{C}$ in air for 8 hours to facilitate fractography inspections. Following heat-tinting, the alloy 600 specimen underwent an air fatigue extension. This process prevents deformation of the SCC surface during post test fracture of the specimen. The higher strength $10.2 \mathrm{~mm}$ alloy X-750 CT specimens, as well as the fatigue extended alloy 600 specimen, were ultimately pulled apart in tension to expose the fracture surfaces. The amount of SCC in each specimen was measured by optical examination using the procedure in ASTM E813.

${ }^{(1)} \mathrm{K}$ validity size requirements are not met for a $25.4 \mathrm{~mm} \mathrm{CT}$ specimen at the "pseudo" stress intensity factor of 65.9 $\mathrm{MPa} \sqrt{m}_{\mathrm{m}}$ (i.e., the specimen is too small). The fact that $\mathrm{K}$ validity is not met under this condition will not be highlighted throughout this document.

${ }^{(2)}$ In a "nitrogen sparge" X-750 AH SCC test $\left(338^{\circ} \mathrm{C}\right)$ the dissolved hydrogen level was estimated at $\sim 0.08 \mathrm{cc} / \mathrm{kg}$ via an Orbisphere hydrogen analyser. 


\section{RESULTS}

\section{SCCGR Analysis}

Electrical potential drop (EPD) indicated immediate SCC growth at the start of all alloy 600 and X-750 condition AH tests. In a large percentage of the X-750 condition HTH tests an appreciable crack growth rate incubation period (i.e., dwell time prior to the establishment of a constant crack growth rate) was noted by EPD (up to 100 hours). Figure 2 provides a representative example of EPD indicated crack growth rate incubation ${ }^{(3)}$. Actual crack extensions were consistently underpredicted by EPD by a factor ranging from 1.5 to 3 . This under-prediction occurs consistently for materials with branched crack fronts and results from an uncracked metal ligament conduction path. EPD would have dramatically over predicted the actual alloy 600 crack extension if material resistivity increases were not taken into consideration. Because of these EPD limitations, reported crack growth rates were determined by dividing the actual destructive examination (DE) intergranular SCC extension by the portion of the test in which cracking occurred (i.e., test duration-incubation time).

It was hypothesized that X-750 HTH SCCGR incubation time would correlate inversely with the crack growth rate. That is, if the environment (in this study hydrogen level) was conducive to accelerate crack growth rates then this environment should also tend to minimize the SCC incubation period. Figure 3 provides a plot of measured X-750 HTH incubation times vs. SCC growth rate (SCCGR) for data generated in this study (i.e., $\sim 0$ to $150 \mathrm{cc} / \mathrm{kg}$ dissolved hydrogen) and some recently conducted tests as a function of $\mathrm{pH}\left(3.8\right.$ to 8.6 at $\left.360^{\circ} \mathrm{C}\right)$ and in heavy water $\left(\mathrm{D}_{2} \mathrm{O}\right)$ with $50 \mathrm{cc} / \mathrm{kg}$ dissolved hydrogen. All of these X-750 HTH tests were conducted at $360^{\circ} \mathrm{C}$ with a stress intensity factor of $\sim 49 \mathrm{MPa} \sqrt{\mathrm{m}}$. Figure 3 illustrates that the X-750 HTH incubation data was comprised of two populations: one group of specimens which had no discernible incubation period for a wide range of crack growth rates and a second set in which the incubation time was weakly correlated with the crack growth rate. Interestingly, a lack of crack growth rate incubation was associated with 4 out of the 5 slowest X-750 HTH crack growth rates. Note the unusually large incubation times of the $\mathrm{X}-750$ samples tested in $\mathrm{D}_{2} \mathrm{O}$ relative to those tested in light water. This observation may be related to the formation of a more perfect passive oxide in $\mathrm{D}_{2} \mathrm{O}^{12}$.

\section{Influence of Hydrogen on SCCGR}

Dissolved hydrogen crack growth rate test results for each of the three materials investigated in this study are summarized in Figures 4, 5, and 6. These figures illustrate the dramatic (up to 7x) effect of dissolved hydrogen concentration on the crack growth rate of the three nickel alloys investigated in this study. Each of these figures includes plots of the measured crack growth rate as a function of (1) the hydrogen level and (2) the electrochemical corrosion potential $(\mathrm{EcP})$ distance away from the nickel to nickel oxide phase transition $\left(\mathrm{EcP}_{\mathrm{Ni} / \mathrm{NiO}}-\mathrm{EcP}\right)$. Since the corrosion potential of nickel alloys behaves as hydrogen electrodes in hydrogenated water ${ }^{9,13}$, this EcP difference reduces simply to the difference of two hydrogen electrodes ${ }^{(4)}$ :

$$
\mathrm{EcP}_{\mathrm{Ni} / \mathrm{NiO}}-\mathrm{EcP}=\Delta \mathrm{EcP}=1000(\mathrm{RT} / 2 \mathrm{~F}) \ln \left(\left[\mathrm{H}_{2}\right]_{\mathrm{test}} /\left[\mathrm{H}_{2}\right]_{\mathrm{Ni} / \mathrm{NiO}}\right) \quad \text {-in mVs- }
$$

In equation (1), $R$ is the gas constant $[8.314 \mathrm{~J} /(\mathrm{mol} \bullet \mathrm{K})], \mathrm{F}$ is Faraday's constant $(96,500 \mathrm{C} / \mathrm{eq}), \mathrm{T}$ is the absolute temperature $(\mathrm{K}),\left[\mathrm{H}_{2}\right]_{\text {test }}$ is the test hydrogen concentration, and $\left[\mathrm{H}_{2}\right]_{\mathrm{NiNiO}}$ is the hydrogen concentration of the $\mathrm{Ni} / \mathrm{NiO}$ phase transition ${ }^{(5)}$ at the given temperature in the same units as $\left[\mathrm{H}_{2}\right]_{\text {test }}$. In these figures, $\Delta \mathrm{EcP}$ was improved

(3) EPD X-750 HTH crack growth rate incubation often appeared as a region of slow growth prior to steady crack growth rate. A significant portion of this slow growth is believed to be an EPD artifact due to small X-750 resistivity changes that were not accounted for in this study.

${ }^{(4)}$ In a Pourbaix diagram the $\mathrm{Ni} / \mathrm{NiO}$ phase transition (single line) and hydrogen electrodes (multiple lines as a function of $\mathrm{H}_{2}$ level) have the same slope. These lines are common at the hydrogen concentration of the $\mathrm{Ni} / \mathrm{NiO}$ phase transition.

(5) 13.8 and $25 \mathrm{cc} / \mathrm{kg}$ were employed as hydrogen concentrations of the nickel/nickel oxide phase transition at $338^{\circ} \mathrm{C}$ and $360^{\circ} \mathrm{C}$, respectively. The $360^{\circ} \mathrm{C}$ value is based upon contact electrical resistance (CER) measurements ${ }^{9}$ and the 
by subtracting the measured specimen to platinum EcP measurement. This modification corrects the specimen EcP for deviations from that of a true hydrogen electrode. This correction becomes appreciable at hydrogen concentrations less than $\sim 5 \mathrm{cc} / \mathrm{kg}^{\text {! }}$.

For each of these three materials, a maximum in crack growth rate occurred in proximity to the $\mathrm{Ni} / \mathrm{NiO}$ phase transition. The maximum SCCGR for alloy X-750 condition HTH coincides almost exactly with the CER measurement of the $\mathrm{Ni} / \mathrm{NiO}$ phase transition, Figure 4 . The maximum SCCGR for $\mathrm{X}-750 \mathrm{AH}$ was $\sim 30 \mathrm{mV}$ into the nickel oxide regime (Figure 5) and maximum for alloy 600 was $\sim 15 \mathrm{mV}$ into the nickel metal regime. Besides the precise location of the maximum susceptible to SCC, with respect to the hydrogen concentration, the magnitude (peak height) and breadth of the effect (peak width) also varied slightly between the three materials. That is, for $\mathrm{X}$ $750 \mathrm{HTH}$, a $5 \mathrm{x}^{(6)}(1.71 \mathrm{mils} / \mathrm{day} / 0.35 \mathrm{mils} / \mathrm{day})$ maximum SCCGR effect was observed and the region in which variation in hydrogen level accelerated the SCC extended over $80 \mathrm{mV}$, Figure 4. For alloys 600 and X-750 AH these values were $3 x, 95 \mathrm{mV}$ and $7 \mathrm{x}, 150 \mathrm{mV}$ respectively, Figures 5 and 6 .

The environmental SCCGR influence of dissolved hydrogen, as expected, was not appreciably influenced by the stress intensity factor (mechanical effect) in this study. As illustrated in Figure 6, the effect of hydrogen level on alloy 600 crack growth (i.e., extent, location and breadth) was not appreciably different at the two stress intensity factor levels of 27.5 and $65.9 \mathrm{MPa} \sqrt{\mathrm{m}}$. The lack of an interaction between the hydrogen functionality and the stress intensity factor is significant. This result indicates that testing to describe the influence of hydrogen level on nickel alloy SCC need not be conducted as a function of the stress state.

\section{Phenomenological Correlations}

A correlation of the observed crack growth rate hydrogen functionality (Figures 4-6) was developed in terms of the fundamental independent parameter $\mathrm{EcP}_{\mathrm{NiNi}}-\mathrm{EcP}(\Delta \mathrm{EcP})$ and a symmetric exponential function as illustrated in equation (2).

$$
\mathrm{SCCGR} / \mathrm{SCCGR}_{\min }=\left(\mathrm{SCCGR}_{\max } / \mathrm{SCCGR}_{\min }\right) \exp \left\{-1 / 2\left[\left(\Delta \mathrm{EcP}+\Delta \mathbf{E c P}_{\mathbf{0}}\right) / \lambda\right]^{2}\right\}
$$

$\mathrm{SCCGR}_{\max } / \mathrm{SCCGR}_{\min }$ is the ratio of the observed maximum to minimum crack growth rate for a given material. This ratio describes the magnitude of the SCCGR dissolved hydrogen level effect for a specific material (e.g., $\sim 5 \mathrm{x}$ for $\mathrm{X}$ $750 \mathrm{HTH}$, see Figure 4). $\Delta \mathbf{E c P}_{\mathbf{0}}$ is a fitted parameter which describes the extent that the maximum crack growth rate is offset from $\Delta \mathrm{EcP}=0$ (i.e., the location of the $\mathrm{Ni} / \mathrm{NiO}$ phase transition). $\lambda$ is another fitting parameter, which describes the breadth of SCCGR vs. $\triangle \mathrm{EcP}$ peaks. Values for $\Delta \mathbf{E c P}_{\mathbf{o}}$ and $\lambda$ were obtained by linear regression analysis of a linearized version of equation (2). Resulting values of $\Delta \mathbf{E c} \mathbf{P}_{\mathbf{0}}$ and $\lambda$ are given below for each material tested. Figure 7 illustrates that these correlations describe the observed nickel alloy dissolved hydrogen crack growth rate functionality.

\begin{tabular}{|l|l|l|l|l|}
\hline Test Condition & SCCGR $_{\max } /$ SCCGR $_{\min }$ & $\Delta \mathbf{E C P}_{\mathbf{0}}$ & $\lambda$ & Range of Applicability ${ }^{(7)}$ \\
\hline $\mathrm{X}-750 \mathrm{HTH} 360^{\circ} \mathrm{C}$ & 4.89 & 3.0 & 20.7 & $-40 \mathrm{mV}<\Delta \mathrm{EcP}<39 \mathrm{mV}$ \\
\hline $\mathrm{X}-750 \mathrm{AH} 338^{\circ} \mathrm{C}$ & 7.19 & 29.9 & 41.8 & $-100 \mathrm{mV}<\Delta \mathrm{EcP}<50 \mathrm{mV}$ \\
\hline $\mathrm{A} 600338^{\circ} \mathrm{C}$ & 2.81 & -8.9 & 35.0 & $-40 \mathrm{mV}<\Delta \mathrm{EcP}<55 \mathrm{mV}$ \\
\hline
\end{tabular}

$338^{\circ} \mathrm{C}$ measurement is based upon CER measurements as well as corrosion coupon data which indicates that 12.5 $\mathrm{cc} / \mathrm{kg} \mathrm{H}_{2}$ is within the $\mathrm{NiO}$ regime and that $20 \mathrm{cc} / \mathrm{kg}$ is within the Ni regime at $338^{\circ} \mathrm{C}$.

(6) If the nitrogen sparge crack growth rate $(0.069 \mathrm{mils} / \mathrm{day}$ at $<0.1 \mathrm{cc} / \mathrm{kg})$ was employed as the minimum observed SCCGR this effect would be $25 \mathrm{x}$. This crack growth rate could not be plotted in Figure $4 \mathrm{~b}$ since measurement of the specimen to $\mathrm{Pt} \mathrm{EcP}$ was not available. This EcP measurement is required at low hydrogen levels $(<5 \mathrm{cc} / \mathrm{kg})$ to correct for deviations from the potential of a hydrogen electrode.

${ }^{(7)}$ The SCCGR effect of dissolved hydrogen level appears to have an appreciable influence in a narrow range of EcP about the $\mathrm{Ni} / \mathrm{NiO}$ phase transition. 
If the location of $\mathrm{Ni} / \mathrm{NiO}$ phase transition is known, equation (2) can be extrapolated to lower temperatures through the assumption that the magnitude of the dissolved hydrogen effect is temperature independent. CER measurements $\left(288\right.$ to $360^{\circ} \mathrm{C}$ ) of the $\mathrm{Ni} / \mathrm{NiO}$ phase transition ${ }^{9}$ were extrapolated to lower temperatures through a two parameter thermodynamic model. The Gibbs free energy $\left(\Delta \mathrm{G}=\Delta \mathrm{H}_{298}-\mathrm{T} \Delta \mathrm{S}_{298}\right)$ of the phase transition reaction $\left(\mathrm{Ni}+\mathrm{H}_{2} \mathrm{O} \rightarrow \mathrm{NiO}+\mathrm{H}_{2}\right)$ is equal to the $-\mathrm{RT} \ln \left(\mathrm{P}_{\mathrm{H} 2}\right)$ where $\mathrm{R}$ and $\mathrm{T}$ are the quantities defined in equation ( 1$)$ and $\mathrm{P}$ is the hydrogen concentration in atmospheres. $\Delta \mathrm{H}_{298}$ and $\Delta \mathrm{S}_{298}$ are the enthalpy and entropy of formation for the phase transition reaction. $\Delta \mathrm{H}_{298}$ and $\Delta \mathrm{S}_{298}$ were empirically determined to be $20.1 \mathrm{~kJ}$ and $0.011 \mathrm{~kJ} / \mathrm{K}$, respectively, by linear regression analysis of the CER data (i.e., plotting $\ln \left(\mathrm{P}_{\mathrm{H} 2}\right)$ vs. 1/T). The resulting thermodynamic correlation for $\mathrm{P}_{\mathrm{H} 2}{ }^{(8)}$ is provided below.

$$
\mathrm{P}_{\mathrm{H} 2}=\exp [-(20,100-11 \mathrm{~T}) / \mathrm{RT}]
$$

Figure 8 presents model predictions for the coolant hydrogen crack growth rate functionality of alloys 600 and $X$ 750 condition $\mathrm{HTH}$ at $288^{\circ} \mathrm{C}$ with $10 \mathrm{cc} / \mathrm{kg}$ dissolved hydrogen based upon the $\mathrm{Ni} / \mathrm{NiO}$ phase transition of $4.7 \mathrm{cc} / \mathrm{kg}$ $\mathrm{H}_{2}$ and the assumptions previously identified. This figure suggests a $2.7 \mathrm{x}$ influence of $10 \mathrm{cc} / \mathrm{kg}$ dissolved hydrogen at the lower temperature of $288^{\circ} \mathrm{C}$. For alloy 600 at $288^{\circ} \mathrm{C}, 10 \mathrm{cc} / \mathrm{kg} \mathrm{H}_{2}$ is close to the SCCGR maximum. For alloy X-750 HTH this condition yields a hydrogen SCCGR effect which is approximately midway between the maximum and the nickel metal region minimum.

\section{General Corrosion Measurement}

A maximum in nickel alloy SCC susceptibility has been observed in a narrow $(\sim 100 \mathrm{mV})$ region near the Ni/NiO phase transition. Increased SCC near an oxide phase transition is often considered a classic sign of a corrosioncontrolled SCC mechanism ${ }^{14}$. Since corrosion is responsible for crack advance in the film rupture oxidation (FRO) mechanism and is believed to be the main source of detrimental hydrogen in a hydrogen assisted crack (HAC) mechanism, both mechanisms as applied to low electrochemical corrosion potential SCC (LPSCC) are essentially corrosion-driven mechanisms ${ }^{(9)}$. Specifically, if either the FRO or the HAC mechanism is the dominant LPSCC mechanism, then metal corrosion should exhibit a maximum in proximity to the $\mathrm{Ni} / \mathrm{NiO}$ phase transition (i.e., the same functionality with respect to aqueous hydrogen concentration as exhibited by LPSCC).

To evaluate this hypothesis, repassivation tests and Electron Spectroscopy Chemical Analysis (ESCA) corrosion measurements were performed as a function of aqueous dissolved hydrogen ${ }^{15}$. Repassivation results could not discern a metal corrosion hydrogen functionality because of a hydrogen oxidation interference. Figure 9 is a summary of the ESCA corrosion film thickness results. ESCA oxide film thickness determinations were made on external surfaces of three alloy X-750 HTH CT specimens tested as part of this study. As illustrated in Figure 9, ESCA average oxide film thickness did not exhibit the same functionality that was observed with SCC with respect to the dissolved hydrogen level. X- $750 \mathrm{HTH} 360^{\circ} \mathrm{C} \mathrm{SCC}$ crack growth is $\sim 20$ times slower under nitrogen sparge test conditions compared to $14 \mathrm{cc} / \mathrm{kg}$ test conditions. However, the nitrogen sparge oxide film thickness was approximately twice as thick as the $14 \mathrm{cc} / \mathrm{kg}$ oxide film (6500 compared to 2820 angstroms).

As noted in Reference (15), the lack of correlation between SCC and bulk oxide film thickness suggests that either: 1) bulk surface corrosion is not an accurate measure of the corrosion kinetics that occurs at the crack tip or 2) the functionality of SCC with respect to the coolant hydrogen level is unrelated to a corrosion functionality. The authors

\footnotetext{
${ }^{(8)} \mathrm{P}_{\mathrm{H} 2}$ is converted to the more common hydrogen concentration units of cc/kg through the use of an applicable Henry's law coefficient. With the approach employed in this analysis to extrapolate $\mathrm{Ni} / \mathrm{NiO}$ phase transitition measurements to lower temperatures all thermodynamic corrections were lumped into $\Delta \mathrm{G}$.

(9) The assertion that corrosion must be the dominant source of detrimental hydrogen if a HAC mechanism is responsible for SCC is supported by the dissolved hydrogen functionality observed in this study. If hydrogen dissolved in the water (i.e., aqueous hydrogen) was the dominant source of detrimental hydrogen in the metal, one would expect SCC susceptibility to increase monotonically with the aqueous hydrogen concentration, rather than exhibiting a maximum in SCC susceptibility
} 
view the former argument as being more likely. Steady state bulk surface corrosion kinetics are typically a function of the square root of time due to a process controlled by diffusion through the protective oxide. In contrast, at a crack tip where periodic rupture can occur the kinetics of initial film formation are likely controlling the corrosion.

\section{Metal Hydrogen Pickup Measurements}

If the HAC mechanism is the dominant LPSCC mechanism, then the buildup of detrimental hydrogen within the material should exhibit the same aqueous hydrogen concentration functionality as observed in LPSCC. In order to investigate this theory, hydrogen concentration measurements have been made on both stressed and unstressed samples of Alloy 600, Alloy X-750 HTH and on high purity nickel. The global hydrogen concentrations were quantified via thermal extraction (i.e., LECO). Results from the LECO measurements are summarized in Figure 10. These results illustrate that the global hydrogen concentration does not follow the same aqueous hydrogen functionality as observed in LPSCC. Consistent with Reference (16), increased absorbed hydrogen levels ${ }^{(10)}$ were not observed in the non-stressed specimens.

The SCC regions of stressed samples were investigated via nuclear reaction anlaysis (NRA), secondary ion mass spectroscopy (SIMS), and LECO measurements. No clear evidence of increased hydrogen concentrations near stress corrosion cracks were observed from these tests. While NRA measurements suggested that locally high ( $100 \mathrm{wt}$. $\mathrm{ppm}$ ) hydrogen concentrations may exist near the SCC surface, the relatively thick oxides (100's of nm), shallow penetration depth of the NRA beam $(\sim 0.5 \mu \mathrm{m})$, and the likelihood of adsorbed water or hydrated oxides on the surface confounded determination of the true hydrogen concentration in the metal. Analysis of the fatigue precrack, $\mathrm{SCC}$, and post-test fracture regions of compact tension specimens of Alloy 600 and X-750 tested in $\mathrm{D}_{2} \mathrm{O}$ via SIMS were also inconclusive. Evidently, the dissolved hydrogen concentrations in these regions are below the $\sim 100 \mathrm{ppm}$ detection limit of the SIMS system employed.

Lastly, sectioning of the SCC region of X-750 HTH compact tension specimens and analysis via LECO did not show any hydrogen enrichment (2-4 wt. ppm), solid circles in Figure 10. Note that this is in contrast to previous work on X-750 and Alloy 600 , in which, locally high hydrogen concentrations ( $\sim 33$ and 83 wt. ppm) have been reported ${ }^{18,19}$ One possible explanation for this discrepancy is that the research that reported elevated hydrogen concentrations near SCC ${ }^{18,19}$, were from constant extension rate (CERT)-type samples rather than a compact tension specimen. It is possible that the CERT specimens contained a greater SCC crack area per volume of hydrogen sample and facilitated the measurements. Additional investigations into the concentration of hydrogen near a stress corrosion crack tip are critical to assessing the viability of the HAC mechanism.

\section{CONCLUSIONS}

1. Testing has identified a dramatic (up to 7x) effect of dissolved hydrogen level on the crack growth rate of nickel alloys. This hydrogen crack growth rate phenomena has been fundamentally described by the extent that the alloy's corrosion potential deviates from the corrosion potential of the $\mathrm{Ni} / \mathrm{NiO}$ phase transition. Crack tip oxides are often of the nickel oxide structure and this corrosion potential difference represents the relative stability of the SCC controlling oxides. A phenomenological model has been developed to extrapolate the hydrogen functionality to lower temperatures.

2. The determination of an accurate thermal activation energy $(Q)$ requires that all fundamental parameters, other than temperature, are maintained constant. The knowledge that the SCC hydrogen functionality is controlled by proximity to the $\mathrm{Ni} / \mathrm{NiO}$ phase transition not the specific aqueous hydrogen level suggests that $\mathrm{SCC}$ thermal activation tests should actually be conducted within a single oxide stability region at all temperatures.

${ }^{(10)}$ Increased absorbed hydrogen levels imply hydrogen values which are greater than would be expected from Sieverts law solubility expectations. 
3. The environmental SCCGR influence of dissolved hydrogen was not appreciably influenced by the stress intensity factor (mechanical effect) in this study. The lack of an interaction between the hydrogen functionality and the stress intensity factor indicates that testing to describe the influence of hydrogen level on nickel alloy SCC need not be conducted as a function of the stress state.

4. The buildup of hydrogen within nickel alloy does not exhibit the same functionality as SCC with respect to the aqueous hydrogen concentration. This result implies that if hydrogen assisted cracking (HAC) is the dominant SCC mechanism, then isolated active path regions (i.e., surface initiation sites or cracks) must be actively corroding and must be the dominant source of elevated detrimental hydrogen fugacities. However, increased hydrogen levels were not measured in the SCC region of X-750 specimens.

5. Alloy X-750 HTH ESCA bulk surface corrosion film thickness did not exhibit the same functionality that was observed with SCC with respect to the hydrogen level. This observation suggests that either:

(1) bulk surface and crack tip corrosion kinetics are different, or

(2) the functionality of SCC with respect to the hydrogen level is unrelated to a corrosion functionality.

\section{ACKNOWLEDGEMENTS}

The following individuals are recognized for their contribution to this effort: Mark Ando for his assistance in test design and diligence in test facility operation and Mike Hanson for his alloy X-750 ESCA analyses.

\section{REFERENCES}

1. D.S. Morton, S.A. Attanasio, J.S. Fish, M.K. Schurman, Corrosion 1999 NACE Conference, Paper 447, 1999.

2. T. Cassagne, F. Vaillant, P. Combrade, Proc. of the Eighth International Symposium on Environmental Degradation of Materials in Nuclear Power Systems-Water Reactors, p.307, 1997.

3. R.S. Pathania, A.R. McIlree, Proc. of the Third International Symposium on Environmental Degradation of Materials in Nuclear Power Systems-Water Reactors, p.551, 1987.

4. T. Magnin, J.M. Boursier, D. Noel, F. Vallant, Proc. of the Sixth International Symposium on Environmental Degradation of Materials in Nuclear Power Systems-Water Reactors, p.669, 1993.

5. G. Economy, P.W. Pement, Corrosion 1989 NACE Conference, Paper 493, 1989.

6. T. Cassagne, A. Gelpi, Proc. of the Sixth International Symposium on Environmental Degradation of Materials in Nuclear Power Systems-Water Reactors, p.679, 1993.

7. N. Totsuka, S. Sakai, N. Nakajima, H. Mitsuda, Corrosion 2000 NACE Conference, Paper 212, 2000.

8. N. Lewis, W.J.S. Yang, J.S. Fish, D.J. Perry, C.D. Thompson, Proc. of the Eighth International Symposium on Environmental Degradation of Materials in Nuclear Power Systems-Water Reactors, p.266, 1997.

9. S.A. Attanasio et al., Proc. of the Tenth International Symposium on Environmental Degradation of Materials in Nuclear Power Systems-Water Reactors, 2001.

10. D.S. Morton, D. Gladding, M.K. Schurman, C.D. Thompson, Proc. of the Eighth International Symposium on Environmental Degradation of Materials in Nuclear Power Systems-Water Reactors, p.387, 1997.

11. C.D. Thompson, D.M. Carey, N.L. Perazzo, Proc. of the Eighth International Symposium on Environmental Degradation of Materials in Nuclear Power Systems-Water Reactors, p.366, 1997.

12. G. Bellanger and J.J. Rameau, Journal of Material Science, Vol. 32, p. 4355, 1997.

13. M.E. Indig and C. Groot, Corrosion, Vol. 25, p. 455, 1969.

14. PM Scott, Proc. of the Ninth International Symposium on Environmental Degradation of Materials in Nuclear Power Systems-Water Reactors, 1999.

15. D.S. Morton, S.A. Attanasio, G.A. Young, Corrosion 2001 NACE Conference, Paper 117, 2001.

16. T. Magnin, F, Foct, O de Bouvier, Proc. of the Ninth International Symposium on Environmental Degradation of Materials in Nuclear Power Systems-Water Reactors, 1999.

17. W.M. Robertson, Metallurgical Trans., Vol. 8a, p. 1709, 1977. 
Figure 1: Effect of Dissolved Hydrogen on X-750 HTH $360^{\circ} \mathrm{C}$ Crack Growth Rate ${ }^{1}$

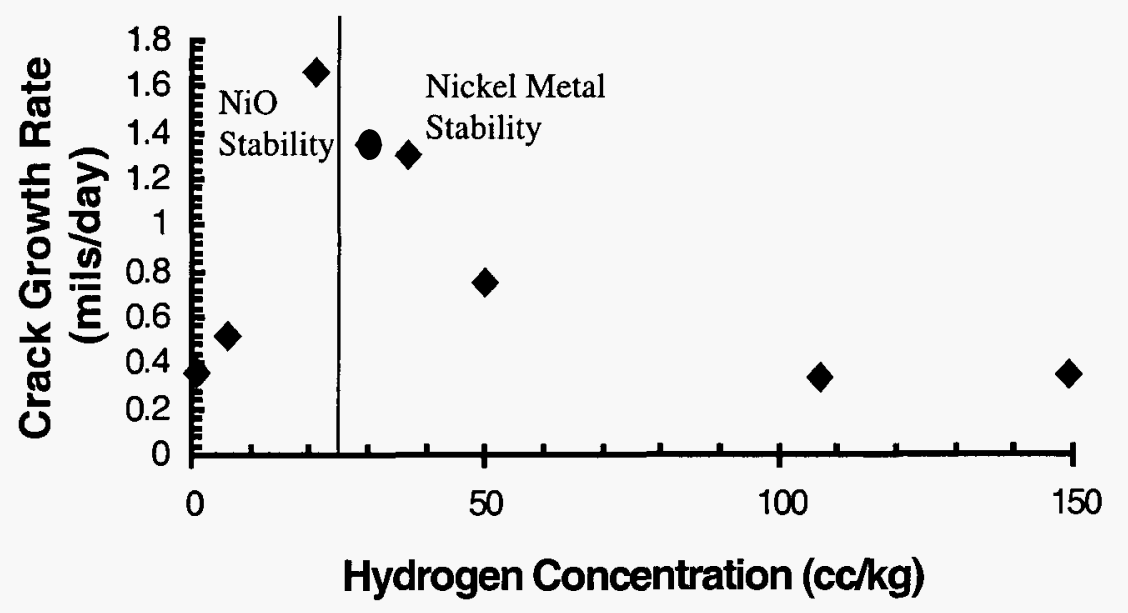

Figure 2: EPD indicated Crack Growth Rate Incubation in a $360^{\circ} \mathrm{C} \mathrm{X-750}$ Condition HTH Test at $10 \mathrm{cc} / \mathrm{kg}$ and $\mathrm{K}=51.5 \mathrm{MPa} V \mathrm{~m}$

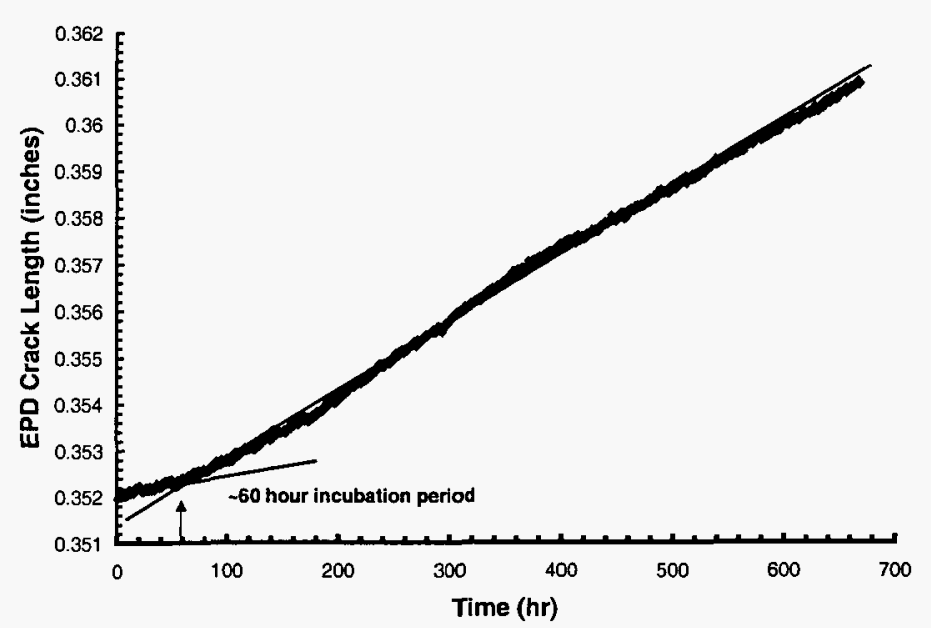

Figure 3: X-750 HTH SCC Incubation Time vs. Crack Growth Rate $\left(360^{\circ} \mathrm{C}, \mathrm{K} 49 \mathrm{MPa} \sqrt{\mathrm{m}}_{\mathrm{m}}\right.$

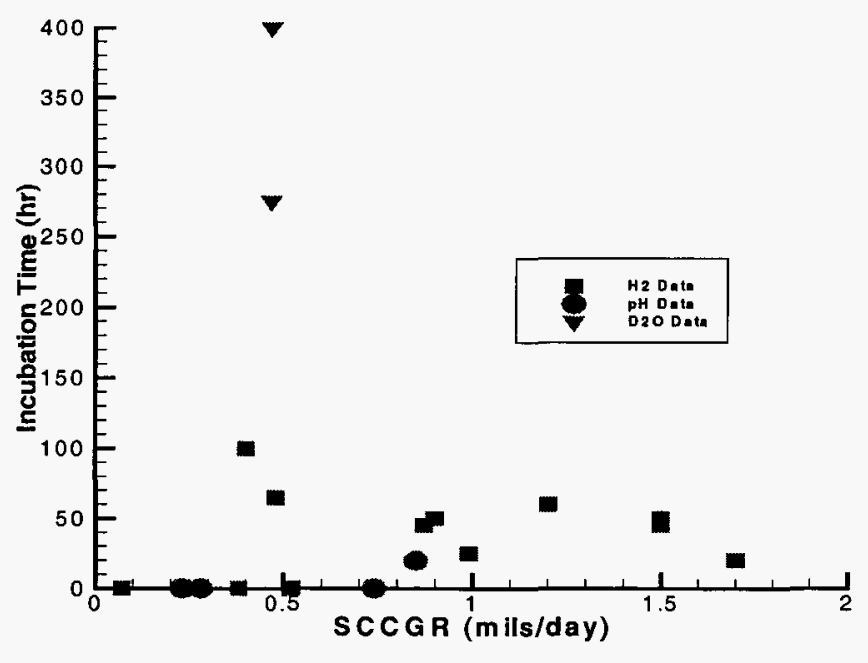


Figure 4: Alloy X-750 Condition HTH Dissolved Hydrogen

Figure 5: Alloy X-750 Condition AH Dissolved Hydrogen Crack Growth Rate Functionality at $338^{\circ} \mathrm{C}$


This point is not shown in the figure below since the specimen to Pt EcP (i.e., deviation from $\mathrm{H}_{2}$ electrode behavior) was not available.
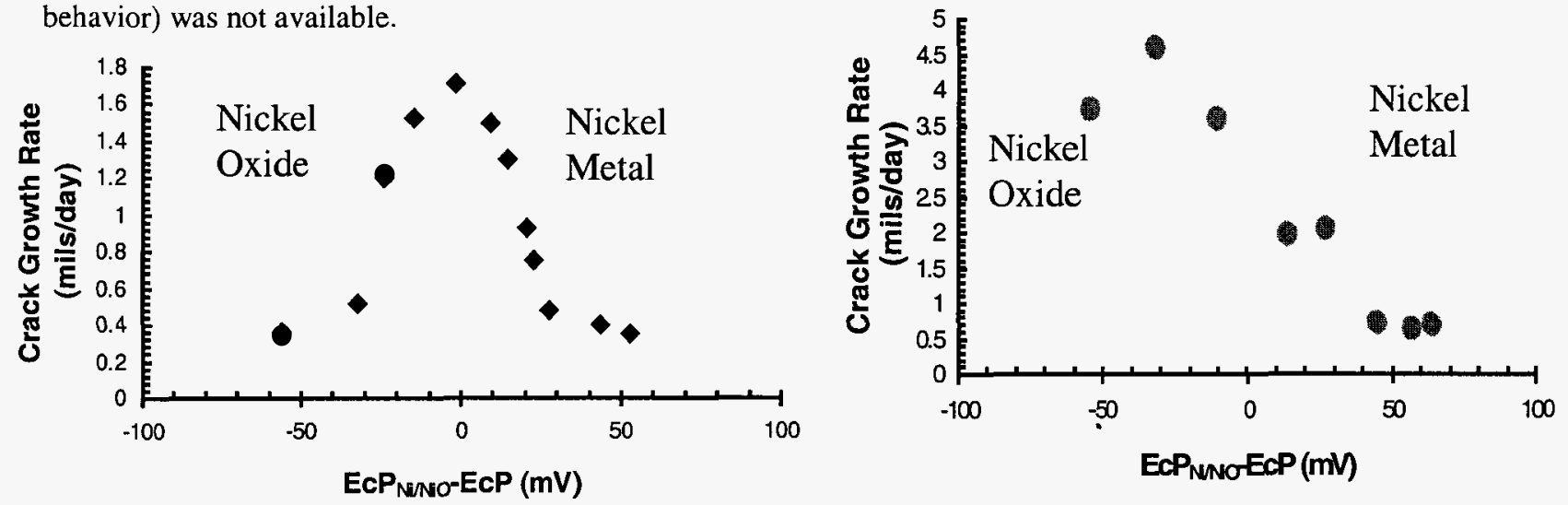

$25 \mathrm{cc} / \mathrm{kg}$ was employed as the hydrogen concentration of the nickel/nickel oxide phase transition at $360^{\circ} \mathrm{C}$. The $360^{\circ} \mathrm{C}$ value is based upon contact electrical resistance (CER) measurements ${ }^{9}$.

$13.8 \mathrm{cc} / \mathrm{kg}$ was employed as the hydrogen concentrations of the nickel/nickel oxide phase transition at $338^{\circ} \mathrm{C}$. This $338^{\circ} \mathrm{C}$ value is based upon CER measurements ${ }^{9}$ as well as corrosion coupon data which indicates that $12.5 \mathrm{cc} / \mathrm{kg}$ hydrogen is within the $\mathrm{NiO}$ regime at $338^{\circ} \mathrm{C}$.

Figure 6: Alloy 600 Dissolved Hydrogen Crack Growth Rate Functionality at $338^{\circ} \mathrm{C}$
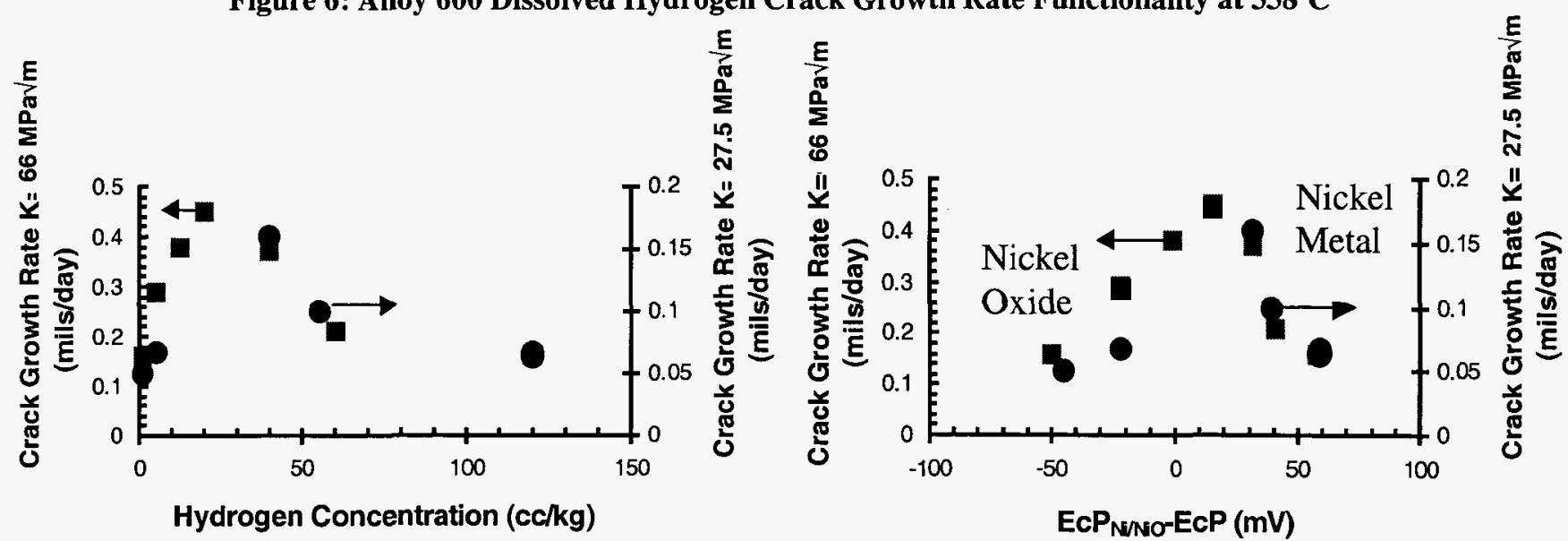

$13.8 \mathrm{cc} / \mathrm{kg}$ was employed as the hydrogen concentrations of the nickel/nickel oxide phase transition at $338^{\circ} \mathrm{C} .13 .8 \mathrm{cc} / \mathrm{kg}$ is based upon CER measurements ${ }^{9}$ as well as corrosion coupon data which indicates that 12.5 $\mathrm{cc} / \mathrm{kg}$ hydrogen is within the $\mathrm{NiO}$ regime at $338^{\circ} \mathrm{C}$. 
Figure 7: Phenomenological Correlation of Alloy X-750 Condition HTH Coolant Hydrogen Crack Growth Rate Functionality at $360^{\circ} \mathrm{C}$

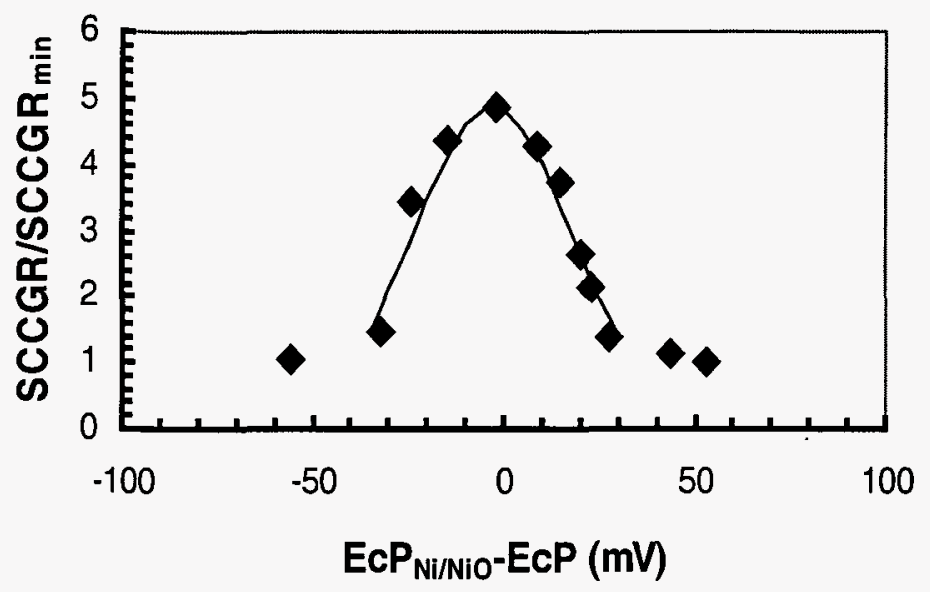

$\mathrm{SCCGR/SCCGR} \mathrm{min}_{\min }=\mathrm{SCCGR}_{\max } / \mathrm{SCCGR}_{\min } \exp \{-$ $\left.1 / 2[(\Delta \mathrm{EcP}+3.0) / 20.7]^{2}\right\}$

$\mathrm{SCCGR}_{\max } / \mathrm{SCCGR}_{\min }=1.71 \mathrm{mils} / \mathrm{day} / 0.35 \mathrm{mils} / \mathrm{day}$

3.0 and 20.7 are fitted parameters

The correlation is valid in the approximate range of $-40 \mathrm{mV}<$ $\Delta \mathrm{EcP}<39 \mathrm{mV}$

Figure 9: Nickel Alloy SCC and ESCA Oxide Film Thickness as a Function of Dissolved Hydrogen Concentration ${ }^{14}$

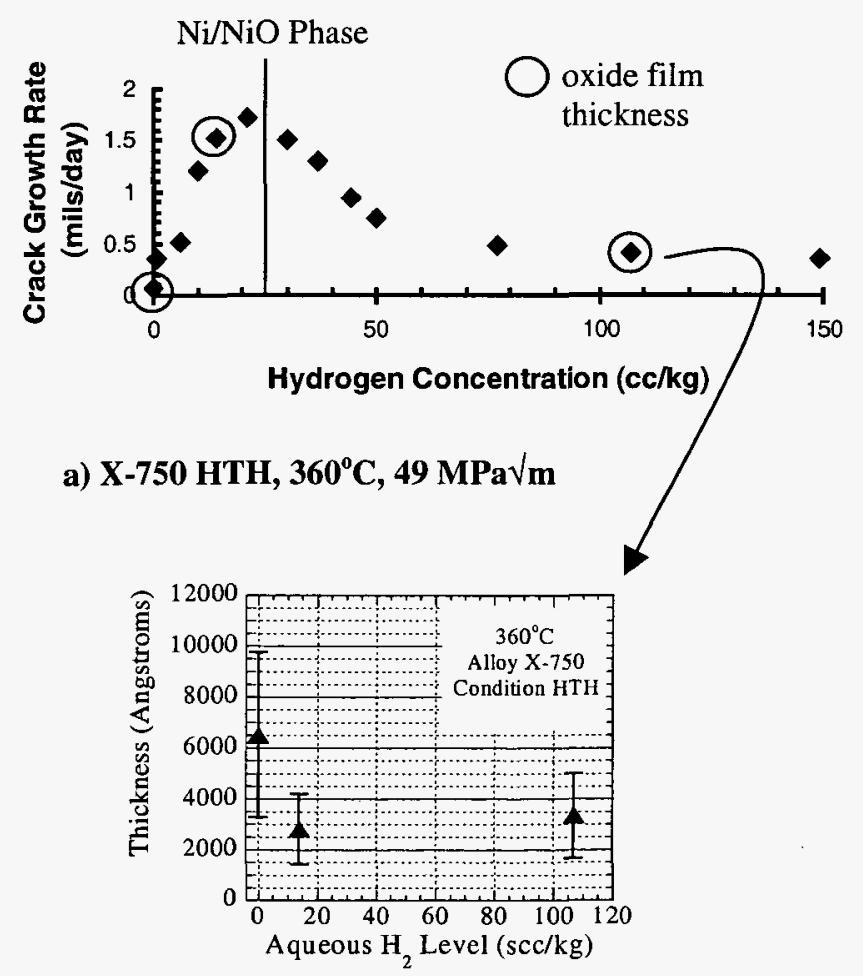

b) $\mathrm{X}-750 \mathrm{HTH}, 360^{\circ} \mathrm{C}, 28$ day ESCA determined oxide film thickness vs. $\mathrm{H}_{2}$
Figure 8: The Effect of Dissolved Hydrogen on Alloys 600 and X-750 HTH SCCGR Extrapolated Down to $288^{\circ} \mathrm{C}$

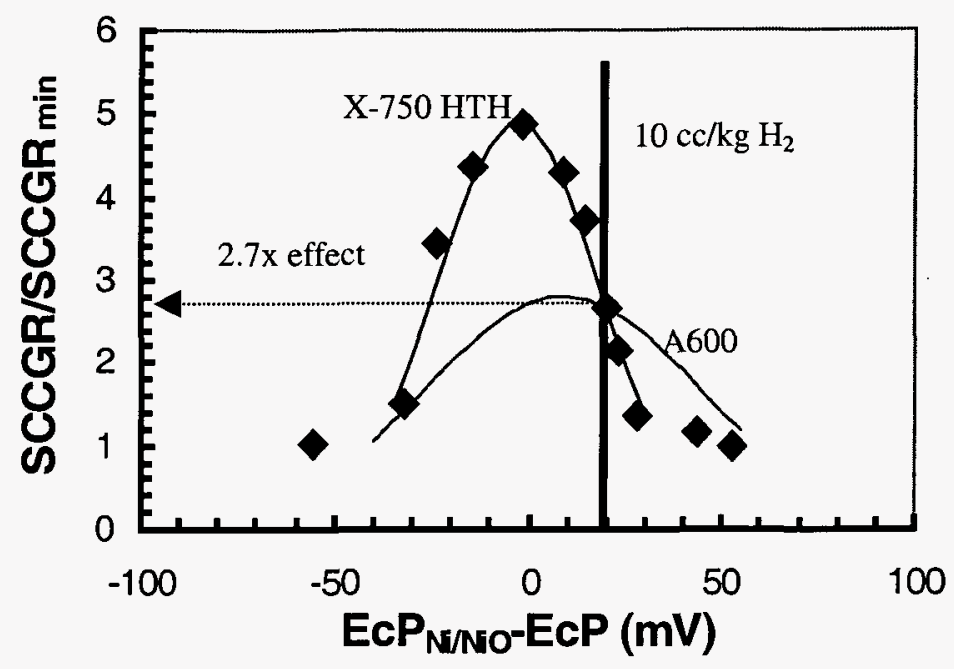

Figure 10: Nickel Alloy Metal Hydrogen Pickup as a Function of Dissolved Hydrogen Concentration

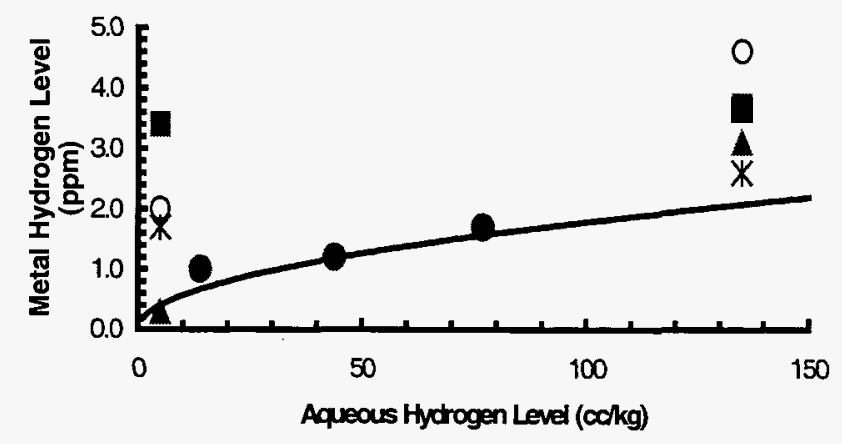

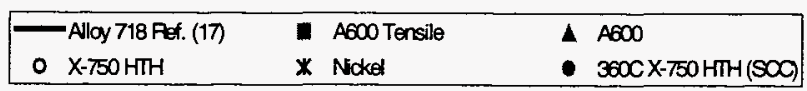

